\title{
Experiencia de capacitación a actores locales en Santiago de Cuba como práctica de innovación social
}

\begin{tabular}{ccc}
\hline Basto, Vivian & Moncada Santos, Margarita & Martínez Tena, Alicia de la C. \\
Universidad de Oriente, Cuba & Universidad de Oriente, Cuba & Universidad de Oriente, Cuba \\
Estrada.vbasto@uo.edu.cu & mmoncada@uo.edu.cu & alicia@uo.edu.cu \\
Hernández Garrido, Margarita V. & Expósito García, Elpidio & Villalón García, Giovanni \\
Universidad de Oriente, Cuba & Universidad de Oriente, Cuba & Universidad de Oriente, Cuba \\
hmargarita@uo.edu.cu & elpidioeg@uo.edu.cu & giovanni@uo.edu.cu \\
Santana González, Yorkys & Castillo Salina, Yander \\
Universidad de Oriente, Cuba & Universidad de Oriente, Cuba \\
yorkys@@uo.edu.cu & yandercs@uo.edu.cu \\
\hline
\end{tabular}

\section{Resumen}

El artículo muestra una estrategia de capacitación a actores locales en el territorio santiaguero en el período 2018-2020 como parte de un resultado del proyecto Fortalecimiento de las capacidades de los actores locales para el logro de la inclusión de grupos vulnerables al desarrollo local del Centro de Estudios Sociales Cubanos y Caribeños Dr. José A. Portuondo de la Universidad de Oriente. A partir del diagnóstico elaborado de las necesidades de capacitación, asesoría y acompañamiento metodológico en el área de las Ciencias Sociales para la labor gubernamental del Consejo de Administración Municipal, se procede a la implementación de las acciones de la estrategia. Se trabaja desde un enfoque de inclusión social de grupos vulnerables al desarrollo local y con la perspectiva integradora del trabajo comunitario integrado y diversas herramientas teórico-metodológicas como la Me- todología de proyecto de desarrollo sociocultural, la educación popular, la comunicación comunitaria y el autodesarrollo participativo. Se experimentan a partir de su aplicación, transformaciones significativas en la gestión de la gobernanza local a diferentes niveles y en diversas dimensiones de la realidad social. La atención a grupos vulnerables en las comunidades por parte de los Delegados de Circunscripciones y Presidentes de Consejos Populares ha devenido en el principal resultado que se muestra. Se une a lo anterior, el fortalecimiento de capacidades, de los actores locales (presidentes de gobierno, de consejos populares y delegados de circunscripciones) para incorporar en sus estrategias, las atenciones a la población en riesgo.

Palabras clave: Innovación social; inclusión social; actores locales; grupos vulnerables; estrategia de capacitación; 


\section{Abstract}

The article shows a strategy for training local actors in the territory of Santiago in the period 2018-2020 as part of a project result Strengthening the capacities of local actors to achieve the inclusion of vulnerable groups in the local development of the Center Cuban and Caribbean Social Studies Dr. José A. Portuondo of the Universidad de Oriente. Based on the elaborated diagnosis of the training, advisory and methodological accompaniment needs in the area of Social Sciences for the governmental work of the Municipal Administration Council, the strategy actions are implemented. We work from an approach of social inclusion of vulnerable groups to local development and with the integrated perspective of integrated community work and various theoretical-methodological tools such as Sociocultural development project methodology, popular education, community communication and participatory self-development. From their application, significant transformations in the management of local governance at different levels and in different dimensions of social reality are experienced. Attention to vulnerable groups in the communities by the Delegates of Circumscriptions and Presidents of Popular Councils has become the main result that is shown. It is added to the above, the strengthening of capacities, of the local actors (presidents of government, of popular councils and delegates of circumscriptions) to incorporate in their strategies, the attentions to the population in risk.
Keywords: Social innovation; social inclusion; local actors; vulnerable groups; training strategy;

\section{INTRODUCCIÓN}

La estrategia que se presenta se desarrolla en el contexto geográfico de los consejos populares Distrito José Martí Norte, José Martí Sur, Los Maceos, El Cristo, Manuel Isla Pérez y Agüero- Marverde, del municipio de Santiago de Cuba. Tiene la intencionalidad de asesorar y capacitar a actores locales en el manejo de herramientas teóricosmetodológicas para el desempeño efectivo de su labor gubernamental; sobre la base del fomento de la participación y la inclusión de grupos vulnerables (Villa, M. (2001) en comunidades santiagueras. La investigación ha sido desarrollada desde la interrogante ¿Cómo fortalecer las capacidades de los actores locales (Presidentes de consejos populares y delegados de circunscripciones) en el fomento de la participación y la inclusión de grupos vulnerables (mujeres, ancianos y jóvenes) en el desarrollo local en comunidades santiagueras?, (Martínez: 2018). 
Acorde a lo planteado en los Lineamientos de la Política Económica y Social, la contribución de las universidades al desarrollo de los territorios, exige de la articulación de recursos teóricos y metodológicos que faciliten el vínculo universidad/sociedad, contribuyendo al mejor desempeño de los gobiernos locales; de ahí que la investigación se plantee como objetivo: Fortalecer las capacidades de los actores locales a través del diseño e implementación de una estrategia de capacitación, que aporte a los gobiernos locales elementos necesarios para la puesta en práctica de acciones que faciliten la inserción de grupos vulnerables al desarrollo local, rebasando los factores condicionantes de la vulnerabilidad social vinculada a la pobreza y la exclusión social.

En las Ciencias Sociales cubanas, existen numerosos experiencias de capacitación a actores del desarrollo local, incluida la participación de las universidades y el accionar de los Centros Universitarios Municipales, sin embargo, ubicar en el centro de la capacitación a los Presidentes de consejos populares y delegados de circunscripción la mirada crítica en torno a la relación vulnerabilidad social/pobreza/exclusión/inclusión social, desde postulados teóricos metodológicos interdisciplinares, dota de novedad científica esta propuesta.

La estrategia se articula en conferencias, cursos, talleres y asesorías y aunque estuvo dirigida fundamentalmente a Presidentes de Consejos Populares y Delegados de Circunscripción, se incorporaron también en algunas acciones a directivos del gobierno local y dirigentes empresariales, especialmente en las temáticas referidas a la dimensión comunicativa, buscando suplir carencias identificadas en tal sentido en ellos.

La investigación tiene como antecedentes los trabajos realizados por el equipo de profesores e investigadores del Centro de Estudios Sociales Cubanos y Caribeños "José Antonio Portuondo" de la Universidad de Oriente desde el año 2011, derivadas de los proyectos "La juventud cubana entre modelos de inclusión y exclusión sociocultu- 
ral: estudio de caso en jóvenes universitarios del oriente cubano", "Modelos de atención a la pobreza en comunidades santiagueras: entre la inclusión y la exclusión sociocultural (2013) y "Fortalecimiento de las capacidades de los actores locales para el logro de la inclusión de grupos vulnerables al desarrollo local" (2016), asociado al Programa Nacional Desarrollo Local y la obtención de dos premios del CITMA y la Academia de Ciencias de Cuba, respectivamente. Participan además en el resultado, los Centros Universitarios Municipales de II Frente y Palma Soriano.

\section{DESARROLLO}

\section{Materiales y/o Método}

La estrategia aplicada para la capacitación de actores locales es presentada como una innovación social que ha generado herramientas $y$ mecanismos para perfeccionar los procesos de la gestión local en el nivel de los Consejos Populares. Ha devenido en una solución en la actualización de los discursos y narrativas de los actores comunitarios en nuevos escenarios de desarrollo $y$ ha fortalecido el marco de valores sociales que caracteriza el hacer de los grupos de trabajo comunitario. Ello facilita el tratamiento a estos conceptos adaptado a las peculiaridades del desarrollo local e incorporado una visión propia que abre mayores posibilidades a su incorporación a la gestión y las políticas públicas el país. Esto dota de novedad científica esta propuesta, consiguiendo mejorar el impacto de su actuar como actores del desarrollo local.

La experiencia se estructura en tres etapas: la 1ra etapa de diagnóstico, tuvo como objetivo caracterizar el nivel de conocimiento de los actores locales para identificar las principales manifestaciones de la vulnerabilidad de determinados grupos sociales respecto a las dimensiones en estudio en las comunidades seleccionadas; la 2 da etapa es de implementación de la estrategia, mediante la ejecución del programa de Conferencias, Cursos, Talleres y Asesorías y la 3ra etapa de Evaluación y control, con el propósito esencial de someter a valoración las acciones ejecutadas y los resultados alcanzados en el proceso de ejecución de la estrate- 
gia. Se capacitan a presidentes de consejos populares y delegados de circunscripciones de cuatro consejos populares del municipio Santiago de Cuba.

Para el desarrollo del diagnóstico se emplearon técnicas de investigación social como la entrevista individual y grupos de discusión. Lo que presupuso desentrañar el vacío epistemológico de los actores en cuestión en torno a temáticas como los estudios de comunidades, el auotodesarrollo participativo, el triple autodiagnóstico participativo, la participación comunitaria, la mediación, negociación; así como sus niveles de conocimiento. Todos estos procesos se interconenctan en la labor de gestión gubernamental y en el área del Trabajo Comunitario Integrado.

La estrategia de capacitación tiene como:

- Misión: Capacitar a los actores locales (Presidentes de Consejos Populares y Delegados de Circunscripción) en los elementos necesarios para incentivar la participación y la inclusión de grupos vulnerables en el desarrollo local en comunidades santiagueras.
- Visión: Los actores locales alcanzan un nivel de preparación que les permita realizar acciones que faciliten la inserción de grupos vulnerables al desarrollo local, especialmente mujeres, ancianos y jóvenes, contribuyendo a rebasar los factores condicionantes de la vulnerabilidad social vinculada a la pobreza y la exclusión social.

Públicos Metas:

- Delegados Circunscripción, Presidentes Consejos Popular, Directivos empresariales, Otros líderes comunitarios.

Objetivo de la estrategia Fortalecer las capacidades de los actores locales en el trabajo por la inserción de grupos vulnerables al Desarrollo Local, especialmente mujeres, ancianos y jóvenes, reconociendo los factores de vulnerabilidad asociados a ellos y rebasando los factores condicionantes de la vulnerabilidad social vinculada a la pobreza y la exclusión social.

Para ello se plantea como Objetivos Específicos:

1. Profundizar en el conocimiento en torno a las principales consideraciones teóricas y 
metodológicas de las Ciencias Sociales cubanas en torno al estudio de la relación vulnerabilidad social/pobreza/exclusión/inclusión social.

2. Aportar herramientas teórico metodológicas que le permitan a los actores locales caracterizar las principales manifestaciones de vulnerabilidad de determinados grupos sociales, especialmente mujeres, ancianos y jóvenes.

3. Apropiarse de herramientas de la comunicación social que les faciliten su interactuar con la población y otros actores locales.

\section{Etapas}

- 1ra etapa: Diagnóstico: (Enerojulio 2018). Tiene como objetivo caracterizar el nivel de conocimiento de los actores locales para identificar las principales manifestaciones de la vulnerabilidad de determinados grupos sociales respecto a las dimensiones en estudio en las comunidades seleccionadas.

- 2da etapa: Implementación de la estrategia. (septiembre 2018-diciembre 2019).

- Ejecución del programa de conferencias, cursos, talleres y asesorías.
- 3ra etapa: Evaluación y control, con el propósito esencial de someter a valoración las acciones ejecutadas y los resultados alcanzados en el proceso de ejecución de la estrategia. (Enero 2018- diciembre 2019).

\section{Principales ejes temáticos}

1. El trabajo comunitario: fundamentos teóricos metodológicos para el tratamiento a las vulnerabilidades sociales.

2. Metodología de proyecto de desarrollo sociocultural comunitario.

3. Vulnerabilidad, exclusión e inclusión social: una mirada interdisciplinar desde las ciencias sociales cubanas.

4. Consideraciones metodológicas en torno a las dimensiones de la vulnerabilidad social.

5. El diagnóstico sociocultural: pautas para su elaboración.

6. La integración de actores locales como elemento del trabajo comunitario.

7. La comunicación social como herramienta del trabajo comunitario en función del desarrollo local.

8. Comunicación social e integración de grupos vulnerables: consideraciones para la acción. 
Experiencia de capacitación a actores locales en Santiago de Cuba..

Plan de acciones

\section{Cursos}

- Fundamentos teóricos metodológicos para el tratamiento a las vulnerabilidades sociales.

- La comunicación efectiva y la mediación de conflictos en el trabajo con jóvenes.

Taller

- Fundamentos teóricos-metodológicos del trabajo de comunidades.

- La educación ambiental comunitaria.

- La atención al adulto mayor desde las iniciativas locales e instituciones.

- La violencia y sus manifestaciones sociales

Entrenamiento

- Metodología para la elaboración de proyectos de desarrollo sociocultural comunitario.

- La Educación popular como herramienta para el desarrollo de comunidades

\section{Conferencias}

- La necesidad de la integración de los actores locales para el desarrollo local.

- Asesoría a presidentes y delegados de todos de los consejos populares (Todo el año).
- Medios y públicos: hacia un nuevo modelo de gestión para la radio en el marco de una política de inclusión social.

- La comunicación comunitaria en el contexto local.

- La relación vulnerabilidad social, alimentación y salud para el desarrollo local.

- Relación entre la identidad nacional, local y comunitaria.

- La participación juvenil a partir del análisis de los consumos culturales juveniles.

\section{RESULTADOS Y DisCUSIÓN}

La implementación de la estrategia de capacitación exigió la caracterización de los actores locales atendiendo a variables sociodemogáficas y a la utilización de la cartografía participativa. Sobre el primero las variables nivel de escolaridad, conocimientos sobre las metodologías para el trabajo comunitario y años de experiencia como Delegados resultaron provechosos sus resultados. Se destaca que un $4 \%$ de delegados y presidentes son Máster, lo que sustenta el dominio de herramientas metodológicas para investigar y transformar las realidades sociales. Las especialidades que se destacan son las de las Ciencias de la Edu- 
cación, así como el área de Salud. Solo un $5 \%$ de los presidentes de consejos populares y un $3 \%$ de los delegados de circunscripciones tienen conocimiento teórico-metodológico del trabajo en comunidades. Más del $50 \%$ ejercen el mandato por vez primera; este dato refuerza la necesidad de la capacitación en herramientas que permitan orientar los diagnósticos, la gestión comunitaria y la comunicación. Una fortaleza se concentra en el dominio de las normativas y procedimientos de la Asamblea Nacional del Poder Popular para el Trabajo Comunitario Integrado. (Asamblea Nacional del Poder Popular, La Habana, 1995).
Sobre la cartografía participativa, se actualizaron las prácticas culturales, los espacios de diálogos comunitarios, tradiciones, como fuentes de datos importantes para le generación de acciones socioculturales.

La estrategia fue presentada a los Presidentes de los Consejos Populares para legitimar su concepción y conocer si los temas propuestos son realmente los que demandan. La realización del III Taller para el trabajo comunitario integrado de la Asamblea Municipal del poder popular, celebrado el pasado año 2018, en Santiago de Cuba, constituyó el principal espacio para la concertación de acciones.

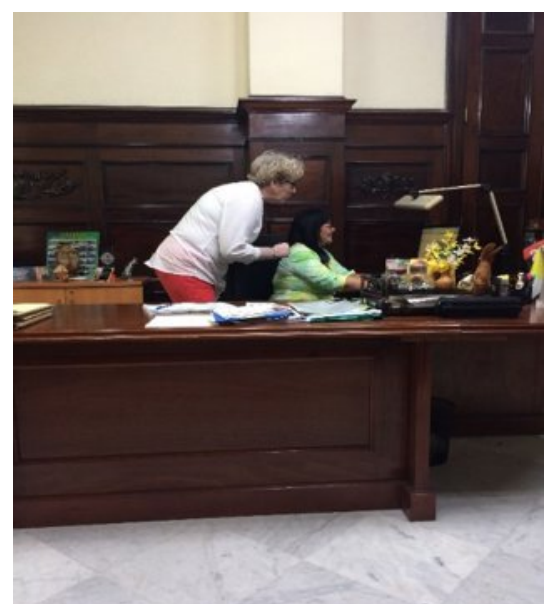

Ilustración.1: La investigadora Alicia Martínez Tena, coordinadora del Grupo Asesor de Trabajo Comunitario Integrado de la Asamblea Provincial del Poder popular Provincial, compartiendo con la Secretaria de Asamblea Provincial del Poder Popular que atiende el Trabajo Comunitario Integrado acerca de la celebración del II taller provincial. 


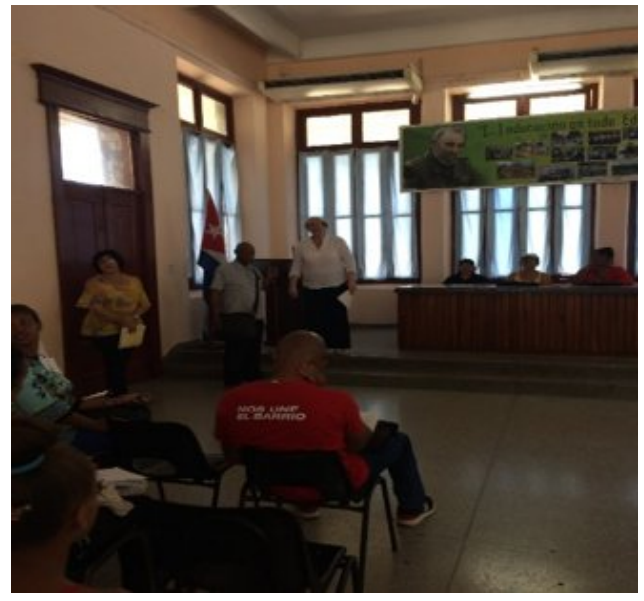

Ilustración 2. Investigadores de la Universidad de Oriente participan en acción de acompañamiento al Gobierno Provincial de Santiago de Cuba.

La estrategia de capacitación es una práctica de innovación social que busca alcanzar resultados en valores sociales, entre ellos, el bienestar, inclusión social, solidaridad, participación, y refuerzan la capacidad de acción sociocomunitaria. Se presenta como un conjunto de servicios (conferencias, asesorías, talleres, entrenamientos), que, en su aplicación, favorece el desarrollo de relaciones de horizontalidad, donde el conocimiento añadido, repercute en las dinámicas locales: iniciativas, creatividad, inclusiones, empoderamiento, valores.

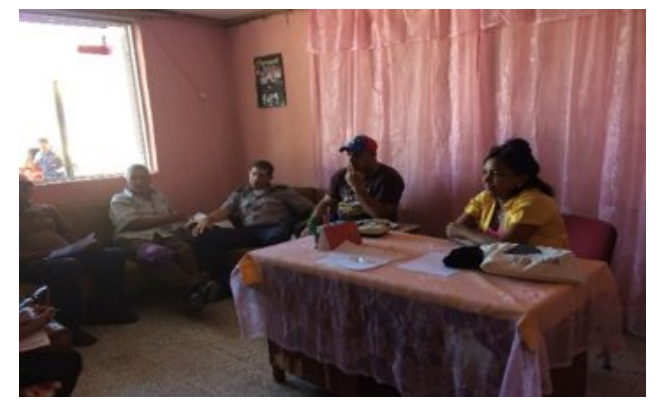

Ilustración 3. La investigadora Vivian Basto Estrada se desempeña como coordinadora del Taller EI Diagnóstico Participativo en el Consejo popular EI Cristo.

Los postulados de partida giran en torno a que más allá de la importancia de la noción o del enfoque (vulnerabilidad, pobreza, marginalidad, exclusión) lo importante es el modelo teórico y empírico de análisis. La vulnerabilidad es un concepto complejo, confluyen aspectos relacionados con los hogares, los individuos, así como las características ambientales, económicas, culturales y políticas de la sociedad". (Cardoso; Martínez, Expósito: 2016, Pp, 747-748)1.

1. Cardoso, J, Expósito, E. \& Martínez, A. (2016). La realidad angolana desde la vulnerabilidad social. Experiencias en comunas rurales en Belas. Revista Santiago, $\mathrm{n}^{\circ}{ }^{141}$, septiembre - diciembre, pp. 745 - 753. 
Desde esta lógica, la combinación de algunas características sociales, económicas, culturales, ambientales, políticas, dentro de un contexto estructural, mediado por las políticas sociales, permiten explicar la emergencia de grupos poblacionales que son socialmente vulnerables. La vulnerabilidad es un concepto dinámico y multidimensional que incluye la exposición de riesgos sociales, culturales y naturales. Los resultados del diagnóstico apuntaron a la presencia de grupos poblacionales en condiciones de riesgos, vulnerables. Capacitar a los distintos actores locales en cómo identificar las principales manifestaciones de vulnerabilidad, su tratamiento conceptual y metodológico y asociar al concepto un grupo de coordenadas constituyó el principal objetivo de la estrategia. Se trabajó con los diferentes tipos de vulnerabilidad: vulnerabilidad sociocultural (accesos a los circuitos de promoción y consumo cultural) vulnerabilidad ambiental (riesgos ante desastres naturales); vulnerabilidad de salud (ante las dinámicas del proceso salud/enfermedad); por violencia en ambientes escolares; por dificultades en el proceso de orientación y formación vocacional y vulnerabilidad según dinámicas y organización familiar. (http://www.vbiobio.cl/cps/ponencial doc/htm)

Se experimenta a partir de la implementación del sistema de acciones una motivación mayor hacia el trabajo en las comunidades. La atención a grupos vulnerables (personas con determinada discapacidad, ancianos, ha aumentado. Los proyectos de desarrollo sociocultural como herramienta de la gestión comunitaria, han comenzado a diversificarse en el mapa sociocultural de los Consejos populares donde ha comenzado a ser aplicada la estrategia, la menos, como intención y voluntad, entre ellos: los proyectos Sin Límite y Herencia infinita; en los consejos populares José Martí Sur y Los Maceos; respectivamente donde se trabaja en la reinserción social de personas en situación de discapacidad y la promoción de valores históricos de la comunidad en los niños. Así mismo se integra el impacto del proyecto Vino de mamá del Consejo Popular Agüero- Marverde. Acciones que trabajan con solidez metodológica 
la recreación sociocultural y la calidad de vida de los moradores. Hasta el momento en que se socializa este resultado, se han capacitado 50 Delegados de Circunscripción y Presidentes de Consejos Populares y disponen de herramientas para el desarrollo de valores sociales, como la solidaridad y la inclusión social.

\section{Conclusiones}

La estrategia de capacitación es una práctica de innovación social, con un grupo de acciones dirigidas a elevar los niveles de gestión participativa por los Delegados de Circunscripción y Presidentes de Consejos Populares para identificar a grupos vulnerables, desarrollar valores como la solidaridad, la inclusión, y generar procesos de participación para el desarrollo local.

Para cada mandato de las gobernanzas locales, es conveniente desarrollar un sistema capacitador sobre la base de necesidades derivadas del diagnóstico de actores y de las comunidades.
Como problema social, la atención a grupos vulnerables para el logro de su inclusión al desarrollo local es una tarea prioritaria para el desarrollo local de los territorios.

La propuesta presentada constituye una herramienta de trabajo eficaz para la capacitación de actores locales en su labor gestión gubernamental.

Con la aplicación de las acciones presentadas, se logra la inclusión de grupos vulnerables al desarrollo local del territorio santiaguero a partir del manejo y aplicación de herramientas teórico-metodológicas que favorecen el desarrollo humano, local y social del municipio Santiago de Cuba.

\section{BIBLIOGRAFÍA}

Cardoso, J, Expósito, E y Martínez, A (2016): La realidad angolana desde la vulnerabilidad social. Experiencias en comunas rurales en Belas. Revista Santiago, no.141, septiembre- diciembre,pp. 745-753.

Ley 91 de los Consejos Populares. 13 julio del 2000. Asamblea Nacional del Poder Popular, La Habana. 
Martínez Tena Alicia de la Caridad (2018). Centro de Estudios Sociales Cubanos y Caribeños Dr. José A. Portuondo. Universidad de Oriente. 2018: La integración de los actores sociales para el trabajo comunitario: Conferencia Magistral II Taller de trabajo Comunitario. Municipio Santiago de Cuba.

Villa, M. (2001). "Vulnerabilidad social: notas preliminares". Trabajo presentado al Seminario Internacional sobre las diferentes expresiones de la vulnerabilidad social en América Latina y el Caribe. CEPAL-CELADE. Santiago de Chile. 